\title{
AN A PRIORI BOUND FOR AUTOMATED MULTI-LEVEL SUBSTRUCTURING
}

\author{
KOLJA ELSSEL* AND HEINRICH VOSS*
} zation

Key words. Eigenvalues, AMLS, substructuring, nonlinear eigenproblem, minmax characteri-

AMS subject classification. $65 \mathrm{~F} 15,65 \mathrm{~F} 50$

Abstract. The Automated Multi-Level Substructuring (AMLS) method has been developed to reduce the computational demands of frequency response analysis and has recently been proposed as an alternative to iterative projection methods like Lanczos or Jacobi-Davidson for computing a large number of eigenvalues for matrices of very large dimension. Based on Schur complements and modal approximations of submatrices on several levels AMLS constructs a projected eigenproblem which yields good approximations of eigenvalues at the lower end of the spectrum. Rewriting the original problem as a rational eigenproblem of the same dimension as the projected problem, and taking advantage of a minmax characterization for the rational eigenproblem we derive an a priori bound for the AMLS approximation of eigenvalues.

1. Introduction. Over the last few years, a new method for frequency response and eigenvalue analysis for complex structures has been developed by Bennighof and co-authors [3], [4], [5], [6], [14] known as Automatic Multi-Level Substructuring (AMLS). Here the large finite element model is recursively divided into very many substructures on several levels based on the sparsity structure of the system matrices. Assuming that the interior degrees of freedom of substructures depend quasistatically on the interface degrees of freedom, and modeling the deviation from quasistatic dependence in terms of a small number of selected substructure eigenmodes the size of the finite element model is reduced substantially yet yielding satisfactory accuracy over a wide frequency range of interest. Recent studies ([16], [14], e.g.) in vibroacoustic analysis of passenger car bodies where very large FE models with more than one million degrees of freedom appear and several hundreds of eigenfrequencies and eigenmodes are needed have shown that AMLS is considerably faster than Lanczos type approaches.

We stress the fact that substructuring does not mean that it is obtained by a domain decomposition of a real structure, but it is understood in a purely algebraic sense, i.e. the dissection of the matrices can be derived by applying a graph partitioner like CHACO [11] or METIS [15] to the matrix under consideration. However, because of its pictographic nomenclature we will use terms like substructure or eigenmode from frequency response problems when introducing the AMLS method.

From a mathematical point of view AMLS is a projection method where the ansatz space is constructed exploiting Schur complements of submatrices and truncation of spectral representations of subproblems. In this paper we will take advantage of the facts that the original eigenproblem is equivalent to a rational eigenvalue problem of the same dimension as the projected problem in AMLS, which can be interpreted as exact condensation of the original eigenproblem with respect to an appropriate basis. Its eigenvalues at the lower end of the spectrum can be characterized as minmax values of a Rayleigh functional of this rational eigenproblem. Hence, comparing the Rayleigh quotient of the projected problem and the Rayleigh functional of the rational

${ }^{*}$ Department of Mathematics, Hamburg University of Technology, D-21071 Hamburg, Germany ( $\{$ elssel, voss $\}$ @tu-harburg.de) 
problem we derive an a priori bound for the error of the AMLS method.

Bekas and Saad [2] for the one level version of AMLS identified the AMLS approximation as linearization of the rational eigenproblem mentioned in the last paragraph which motivated them to suggest three modifications of AMLS, a second order approximation, expanding the projection space by Krylov subspaces, and a combination of these two modifications.

In a recent paper Yang et al. [21] considered a one level version of AMLS. The authors obtained a simple heuristic for choosing spectral components from each substructure suggesting to drop all eigenpairs $(\omega, \phi)$ of substructures in the reduction process such that

$$
\rho_{1}(\omega):=\frac{\lambda_{1}}{\omega-\lambda_{1}} \leq \tau
$$

where $\lambda_{1}$ is the smallest eigenvalue of the problem under consideration, and $\tau$ is a given tolerance. By our new a priori bound this omission rule guarantees that the relative error of the smallest eigenvalue of the projected problem is not greater than the tolerance $\tau$.

Our presentation is organized as follows. Section 2 gives a brief overview of the automatic multi-level substructuring and derives the exactly condensed rational eigenproblems, which is equivalent to the original eigenproblem. Section 3 collects the variational characterization of nonlinear and nonoverdamped eigenvalue problems which are exploited in Section 4 to deduce a priori bounds for the component mode method, the one level version of AMLS, and the general automated multi-level substructuring method. The paper closes with numerical examples in Section 5.

2. Substructuring of eigenproblems. We are concerned with the linear eigenvalue problem

$$
K x=\lambda M x
$$

where $K \in \mathbb{R}^{n \times n}$ and $M \in \mathbb{R}^{n \times n}$ are symmetric and positive definite matrices. We recall that the terms structure, substructure, interface and domain are meant in the algebraic sense to follow.

We first consider one level versions of substructuring methods. Assume that the joint graph of the matrices $K$ and $M$ is partitioned into $r$ substructures such that the rows and columns of $K$ can be reordered in the following way:

$$
K=\left(\begin{array}{ccccc}
K_{\ell \ell 1} & O & \ldots & O & K_{\ell i 1} \\
O & K_{\ell \ell 2} & \ldots & O & K_{\ell i 2} \\
\vdots & \vdots & \ddots & \vdots & \vdots \\
O & O & \ldots & K_{\ell \ell r} & K_{\ell i r} \\
K_{i \ell 1} & K_{i \ell 2} & \ldots & K_{i \ell r} & K_{i i}
\end{array}\right),
$$

and $M$ after reordering has the same block form. Here $K_{\ell \ell j}, j=1, \ldots, r$ is the local stiffness matrix corresponding to the $j$-th substructure, $i$ denotes the set of interface vertices, and $K_{\ell i j}$ describes the interaction of the interface degrees of freedom and the $j$-th substructure.

Distinguishing only between local and interface degrees of freedom $K$ and $M$ have the following form:

$$
K=\left(\begin{array}{ll}
K_{\ell \ell} & K_{\ell i} \\
K_{i \ell} & K_{i i}
\end{array}\right) \quad \text { and } \quad M=\left(\begin{array}{ll}
M_{\ell \ell} & M_{\ell i} \\
M_{i \ell} & M_{i i}
\end{array}\right) .
$$


We transform the matrix $K$ to block diagonal form using block Gaussian elimination, i.e. we apply the congruent transformation with

$$
P=\left(\begin{array}{cc}
I & -K_{\ell \ell}^{-1} K_{\ell i} \\
0 & I
\end{array}\right)
$$

to the pencil $(K, M)$ obtaining the equivalent pencil

$$
\left(P^{T} K P, P^{T} M P\right)=\left(\left(\begin{array}{cc}
K_{\ell \ell} & 0 \\
0 & \tilde{K}_{i i}
\end{array}\right),\left(\begin{array}{cc}
M_{\ell \ell} & \tilde{M}_{\ell i} \\
\tilde{M}_{i \ell} & \tilde{M}_{i i}
\end{array}\right)\right) .
$$

Here $K_{\ell \ell}$ and $M_{\ell \ell}$ stay unchanged, and

$$
\begin{aligned}
\tilde{K}_{i i} & =K_{i i}-K_{\ell i}^{T} K_{\ell \ell}^{-1} K_{\ell i} \text { is the Schur complement of } K_{\ell \ell} \\
\tilde{M}_{\ell i} & =M_{\ell i}-M_{\ell \ell} K_{\ell \ell}^{-1} K_{\ell i}=\tilde{M}_{i \ell}^{T} \\
\tilde{M}_{i i} & =M_{i i}-M_{i \ell} K_{\ell \ell}^{-1} K_{\ell i}-K_{i \ell} K_{\ell \ell}^{-1} M_{\ell i}+K_{i \ell} K_{\ell \ell}^{-1} M_{\ell \ell} K_{\ell \ell}^{-1} K_{\ell i} .
\end{aligned}
$$

Neglecting in (2.3) all rows and columns corresponding to local degrees of freedom, i.e. projecting problem (2.1) to the subspace spanned by columns of $\left(\begin{array}{c}-K_{\ell \ell}^{-1} K_{\ell i} \\ I\end{array}\right)$ one obtains the method of static condensation

$$
\tilde{K}_{i i} y=\lambda \tilde{M}_{i i} y
$$

introduced by Guyan [10] and Irons [13]. For vibrating structures this means that the interior degrees of freedom are assumed to depend quasistatically on the interface degrees of freedom, and the inertia forces of the substructures are neglected.

To model the deviation from quasistatic behavior thereby improving the approximation properties of static condensation we consider the eigenvalue problem

$$
K_{\ell \ell} \Phi=M_{\ell \ell} \Phi \Omega, \quad \Phi^{T} M_{\ell \ell} \Phi=I
$$

where $\Omega$ is a diagonal matrix containing the eigenvalues.

Changing the basis for the local degrees of freedom to a modal one, i.e. applying the further congruent transformation $\operatorname{diag}\{\Phi, I\}$ to problem (2.3) one gets

$$
\left(\left(\begin{array}{cc}
\Omega & 0 \\
0 & \tilde{K}_{i i}
\end{array}\right),\left(\begin{array}{cc}
I & \Phi^{T} \tilde{M}_{\ell i} \\
\tilde{M}_{i \ell} \Phi & \tilde{M}_{i i}
\end{array}\right)\right)
$$

In structural dynamics (2.6) is called Craigh-Bampton form of the eigenvalue problem (2.1) corresponding to the partitioning (2.2). In terms of linear algebra it results from block Gaussian elimination to reduce $K$ to block diagonal form, and diagonalization of the block $K_{\ell \ell}$ using a spectral basis.

Selecting some eigenmodes of problem (2.5) (usually the ones according to eigenvalues which do not exceed a cut off threshold, however, in a recent paper Bai and Lia [1] suggested a different choice based on a moment-matching analysis), and dropping the rows and columns in (2.6) corresponding to the other modes one arrives at the component mode synthesis method (CMS) introduced by Hurty [12] and Craigh and Bampton [7]. Hence, if the diagonal matrix $\Omega_{1}$ contains in its diagonal the eigenvalues to drop and $\Phi_{1}$ the corresponding eigenvectors, and if $\Omega_{2}$ and $\Phi_{2}$ contain the 
eigenvalues and eigenvectors to keep, respectively, then the eigenproblem (2.6) can be rewritten as

$$
\left(\begin{array}{ccc}
\Omega_{1} & 0 & 0 \\
0 & \Omega_{2} & 0 \\
0 & 0 & \tilde{K}_{i i}
\end{array}\right)\left(\begin{array}{l}
x_{1} \\
x_{2} \\
x_{3}
\end{array}\right)=\lambda\left(\begin{array}{ccc}
I & 0 & \tilde{M}_{\ell i 1} \\
0 & I & \tilde{M}_{\ell i 2} \\
\tilde{M}_{i \ell 1} & \tilde{M}_{i \ell 2} & \tilde{M}_{i i}
\end{array}\right)\left(\begin{array}{l}
x_{1} \\
x_{2} \\
x_{3}
\end{array}\right)
$$

with

$$
\tilde{M}_{\ell i j}=\Phi_{j}^{T}\left(M_{\ell i}-M_{\ell \ell} K_{\ell \ell}^{-1} K_{\ell i}\right)=\tilde{M}_{i \ell j}^{T}, j=1,2
$$

and the CMS approximations to the eigenpairs of (2.1) are obtained from the reduced eigenvalue problem

$$
\left(\begin{array}{cc}
\Omega_{2} & 0 \\
0 & \tilde{K}_{i i}
\end{array}\right) y=\lambda\left(\begin{array}{cc}
I & \tilde{M}_{\ell i 2} \\
\tilde{M}_{i \ell 2} & \tilde{M}_{i i}
\end{array}\right) y
$$

In Section 4 we shall prove an a priori bound for the relative error of problem (2.8) taking advantage of the fact that the eigenvalues of the original problem (2.1) are eigenvalues of a rational eigenproblem of the same dimension as the reduced problem (2.8), and that the eigenvalues of the rational problem at the lower end of the spectrum are minmax values of a Rayleigh functional.

If $\lambda$ is not a diagonal entry of $\Omega_{1}$ then the first equation of (2.7) yields

$$
x_{1}=\lambda\left(\Omega_{1}-\lambda I\right)^{-1} \tilde{M}_{\ell i 1} x_{3},
$$

and $\lambda$ is an eigenvalue of (2.1) if and only if it is an eigenvalue of the rational eigenproblem

$$
\begin{aligned}
\left(\begin{array}{cc}
\Omega_{2} & 0 \\
0 & \tilde{K}_{i i}
\end{array}\right)\left(\begin{array}{l}
x_{2} \\
x_{3}
\end{array}\right)=\lambda & \left(\begin{array}{cc}
I & \tilde{M}_{\ell i 2} \\
\tilde{M}_{i \ell 2} & \tilde{M}_{i i}
\end{array}\right)\left(\begin{array}{l}
x_{2} \\
x_{3}
\end{array}\right) \\
& +\lambda^{2}\left(\begin{array}{c}
0 \\
\tilde{M}_{i \ell 1}
\end{array}\right)\left(\Omega_{1}-\lambda I\right)^{-1}\left(\begin{array}{ll}
0 & \tilde{M}_{\ell i 1}
\end{array}\right)\left(\begin{array}{l}
x_{2} \\
x_{3}
\end{array}\right)
\end{aligned}
$$

The number of interface degrees of freedom may still be very large, and therefore the dimension of the reduced problem (2.8) may be very high. It can be reduced further by modal reduction of the interface degrees of freedom in the following way: Considering the eigenvalue problem

$$
\tilde{K}_{i i} \Psi=\tilde{M}_{i i} \Psi \Gamma, \Psi^{T} \tilde{K}_{i i} \Psi=\Gamma, \Psi^{T} \tilde{M}_{i i} \Psi=I,
$$

and applying the congruent transformation to the pencil in (2.6) with $\tilde{P}=\operatorname{diag}\{I, \Psi\}$, we obtain the equivalent pencil

$$
\left(\left(\begin{array}{cc}
\Omega & O \\
O & \Gamma
\end{array}\right),\left(\begin{array}{cc}
I & \hat{M}_{\ell i} \\
\hat{M}_{\ell i}^{T} & I
\end{array}\right)\right)
$$

with

$$
\hat{M}_{\ell i}=\Phi^{T}\left(M_{\ell i}-M_{\ell \ell} K_{\ell \ell}^{-1} K_{\ell i}\right) \Psi=\hat{M}_{i \ell}^{T}
$$

Selecting eigenmodes of (2.5) and of (2.10) and neglecting rows and columns in (2.11) which correspond to the other modes one gets a reduced problem which is the 
one level version of the automated multilevel substructuring method, introduced by Bennighof in [5].

Similarly as for the CMS method we partition the matrices $\Gamma$ and $\Psi$ into

$$
\Gamma=\left(\begin{array}{cc}
\Gamma_{1} & 0 \\
0 & \Gamma_{2}
\end{array}\right) \quad \text { and } \quad \Psi=\left(\Psi_{1}, \Psi_{2}\right)
$$

and rearranging the rows and columns beginning with the modes corresponding to $\Phi_{1}$ and $\Psi_{1}$ to be dropped followed by the ones corresponding to $\Phi_{2}$ and $\Psi_{2}$ problem (2.11) obtains the form

$$
\left(\left(\begin{array}{cccc}
\Omega_{1} & 0 & 0 & 0 \\
0 & \Gamma_{1} & 0 & 0 \\
0 & 0 & \Omega_{2} & 0 \\
0 & 0 & 0 & \Gamma_{2}
\end{array}\right),\left(\begin{array}{cccc}
I & \hat{M}_{12} & 0 & \hat{M}_{14} \\
\hat{M}_{21} & I & \hat{M}_{23} & 0 \\
0 & \hat{M}_{32} & I & \hat{M}_{34} \\
\hat{M}_{41} & 0 & \hat{M}_{43} & I
\end{array}\right)\right)
$$

where

$$
\begin{aligned}
& \hat{M}_{12}=\Phi_{1}^{T}\left(M_{\ell i}-M_{\ell \ell} K_{\ell \ell}^{-1} K_{\ell i}\right) \Psi_{1}=\hat{M}_{21}^{T} \\
& \hat{M}_{14}=\Phi_{1}^{T}\left(M_{\ell i}-M_{\ell \ell} K_{\ell \ell}^{-1} K_{\ell i}\right) \Psi_{2}=\hat{M}_{41}^{T} \\
& \hat{M}_{32}=\Phi_{2}^{T}\left(M_{\ell i}-M_{\ell \ell} K_{\ell \ell}^{-1} K_{\ell i}\right) \Psi_{1}=\hat{M}_{23}^{T} \\
& \hat{M}_{34}=\Phi_{2}^{T}\left(M_{\ell i}-M_{\ell \ell} K_{\ell \ell}^{-1} K_{\ell i}\right) \Psi_{2}=\hat{M}_{43}^{T} .
\end{aligned}
$$

The one level approximations of AMLS to eigenpairs are obtained from

$$
\left(\begin{array}{cc}
\Omega_{2} & 0 \\
0 & \Gamma_{2}
\end{array}\right) y=\lambda\left(\begin{array}{cc}
I & \hat{M}_{34} \\
\hat{M}_{43} & I
\end{array}\right) y .
$$

Similarly as in (2.9) for the CMS method the variables corresponding to the leading two rows of (2.13) can be eliminated yielding a rational eigenproblem

$$
\begin{aligned}
\left(\begin{array}{cc}
\Omega_{2} & 0 \\
0 & \gamma_{2}
\end{array}\right) y= & \lambda\left(\begin{array}{cc}
I & \hat{M}_{34} \\
\hat{M}_{43} & I
\end{array}\right) y \\
& +\lambda^{2}\left(\begin{array}{cc}
0 & \hat{M}_{32} \\
\hat{M}_{41} & 0
\end{array}\right)\left(\begin{array}{cc}
\Omega_{1}-\lambda I & -\lambda \hat{M}_{12} \\
-\lambda \hat{M}_{12} & \Gamma_{1}-\lambda I
\end{array}\right)^{-1}\left(\begin{array}{cc}
0 & \hat{M}_{14} \\
\hat{M}_{32} & 0
\end{array}\right) y(2.1
\end{aligned}
$$

which is equivalent to $(2.1)$.

For the special eigenvalue problem $A x=\lambda x$ the one level approximation (2.14) was identified by Bekas and Saad [2] as linearization of the rational problem (2.15) (which is trivial using the representations (2.13), (2.14), and (2.15)). This was the starting point for further improvements adding a second order approximation, expanding the projection space by Krylov subspaces, and a combination of these two modifications. However, we doubt that these variants will be useful for the general AMLS method.

The general version of AMLS introduced by Bennighof [5] starts with the transformed eigenproblem (2.3), and reduces the dimension taking advantage of the spectral decomposition (2.10). Neglecting eigenvectors corresponding to eigenvalues exceeding a given cut off bound one obtains a reduced pencil on the first level

$$
\left(\left(\begin{array}{cc}
K_{\ell \ell} & 0 \\
0 & \Gamma_{1}
\end{array}\right),\left(\begin{array}{cc}
M_{\ell \ell} & \tilde{M}_{\ell i} \Psi_{1} \\
\Psi_{1}^{T} \tilde{M}_{i \ell} & I
\end{array}\right)\right)
$$


Here the dimension of $K_{\ell \ell}$, i.e. the substructures on the coarsest level usually will be very large. Therefore, one avoids to reduce the problem applying the spectral decomposition (2.5) but one dissects the substructures of the first level again, and applies the same reduction step to the upper left part of the matrices in (2.16).

Assume that the initial substructures are dissected a second time, and rearrange the rows and columns of (2.16) such that the local degrees of freedom of the substructuring on the second level appear first, followed by the newly generated interface degrees of freedom. Then (2.16) obtains the following form

$$
\left(\begin{array}{ccc}
K_{\ell \ell}^{(1)} & K_{\ell i}^{(1)} & 0 \\
K_{i \ell}^{(1)} & K_{i i}^{(1)} & 0 \\
0 & 0 & \Gamma_{1}
\end{array}\right) z=\lambda\left(\begin{array}{ccc}
M_{\ell \ell}^{(1)} & M_{\ell i}^{(1)} & M_{\ell i 1} \Psi_{1} \\
M_{i \ell}^{(1)} & M_{i i}^{(1)} & M_{\ell i 2} \Psi_{1} \\
\Psi_{1}^{T} M_{i \ell 1} & \Psi_{1}^{T} M_{i \ell 2} & I
\end{array}\right) z
$$

Block diagonalizing the rearranged representation $K^{(1)}$ of $K_{\ell \ell}$ by the congruent transformation

$$
\left(\begin{array}{ccc}
I & -\left(K_{\ell \ell}^{(1)}\right)^{-1} K_{\ell i}^{(1)} & O \\
O & I & O \\
O & O & I
\end{array}\right)
$$

yields

$$
\left(\begin{array}{ccc}
K_{\ell \ell}^{(1)} & O & O \\
O & \tilde{K}_{i i}^{(1)} & 0 \\
0 & 0 & \Gamma_{1}
\end{array}\right) z=\lambda\left(\begin{array}{ccc}
M_{\ell \ell}^{(1)} & \tilde{M}_{\ell i}^{(1)} & M_{\ell i 1} \Psi_{1} \\
\tilde{M}_{i \ell}^{(1)} & \tilde{M}_{i i}^{(1)} & \tilde{M}_{\ell i 2} \Psi_{1} \\
\Psi_{1}^{T} M_{i \ell 1} & \Psi_{1}^{T} \tilde{M}_{i \ell 2} & I
\end{array}\right) z
$$

which is reduced considering only the share of the spectral decomposition of

$$
\tilde{K}_{i i}^{(1)} \Psi=\tilde{M}_{i i}^{(1)} \Psi \Gamma, \Psi^{T} \tilde{M}_{i i} \Psi=I,
$$

which correspond to eigenvalues less than the given cut off threshold. Hence we obtain the reduced problem on the second level

$$
\left(\begin{array}{ccc}
K_{\ell \ell}^{(1)} & O & O \\
O & \Gamma_{2} & O \\
O & O & \Gamma_{1}
\end{array}\right) z=\lambda\left(\begin{array}{ccc}
M_{\ell \ell}^{(1)} & \tilde{M}_{\ell i 1}^{(1)} \Psi_{2} & M_{\ell i 1} \Psi_{1} \\
\Psi_{2}^{T} \tilde{M}_{i \ell 1}^{(1)} & I & \Psi_{2}^{T} \tilde{M}_{\ell i 2} \Psi_{1} \\
\Psi_{1}^{T} M_{i \ell 1} & \Psi_{1}^{T} \tilde{M}_{i \ell 2} \Psi_{2} & I
\end{array}\right) z
$$

Continuing with substructuring on the current level, block Gauss eliminating the off diagonal blocks in the left upper block, and reducing the dimension of the current interface block by spectral truncation we finally arrive on a level $p$ where the eigenproblems corresponding to the individual substructures are small enough to be solved by a standard eigensolver. In this situation we apply a final eigenfrequency truncation to the pencil $\left(K_{\ell \ell}^{(p)}, M_{\ell \ell}^{(p)}\right)$ in the left upper corner and receive the projected eigenproblem of AMLS.

We presented here a top-down version of AMLS starting with a spectral truncation of the Schur complement of the local degrees of freedom corresponding to the coarsest substructuring of the problem because this will be convenient in Section 4 when deriving an a priori bound. For an implementation of AMLS this top-down version is inappropriate, because in the first step we need an LU decomposition of the diagonal blocks of $K_{\ell \ell}$ the dimensions of which usually will be very large. 
The equivalent bottom-up version (cf. [3], [4], [5], [9], [14]) starts by generating the substructuring on several levels taking advantage of the joint graph of $K$ and $M$ only, but not of the entries of $K$ and $M$. Each substructure on the finest level is transformed to its quasistatic/modal representation, i.e. we apply the congruent transformation to the restriction of the pencil (2.1) to the local degrees of freedom of the substructure under consideration and those interface variables connected to this substructure which diagonalizes the $K$ part of the restricted pencil, and we reduce the dimension by spectral truncation which is quite inexpensive because these substructures are very small.

Once lowest level substructures have been transformed they are assembled together to form 'parent substructures' on the next level. For each of these substructures we identify the newly generated local degrees of freedom (which were interface ones on the lowest level), and assemble the corresponding local matrices. Again these matrices are small, and block diagonalization and spectral reduction again are inexpensive. Continuing this way assembling to form higher level substructures, and transforming to quasistatic/modal representation, we finally arrive at a model of the entire structure on the coarsest level where we execute a final spectral reduction. It is obvious that this form of AMLS has a very high parallelization potential.

3. Variational characterization of eigenvalues of nonlinear eigenproblems. We consider the nonlinear eigenvalue problem

$$
T(\lambda) x=0
$$

where $T(\lambda) \in \mathbb{R}^{n \times n}$ is a family of real symmetric matrices for every $\lambda$ in an open real interval $J$ which may be unbounded.

For a linear symmetric problem $K x=\lambda M x$ all eigenvalues are real, and if they are ordered by magnitude regarding their multiplicity $\lambda_{1} \leq \lambda_{2} \leq \cdots \leq \lambda_{n}$ then it is well known that they can be characterized by the minmax principle of Poincaré

$$
\lambda_{k}=\min _{V \in S_{k}} \max _{x \in V, x \neq 0} \frac{x^{T} K x}{x^{T} M x}, \quad k=1,2, \ldots, n,
$$

where $S_{k}$ denotes the set of all $k$ dimensional subspaces of $\mathbb{R}^{n}$.

Similar results hold for certain nonlinear eigenvalue problems, too. We assume that the function $f(\lambda, x):=x^{T} T(\lambda) x$ is continuously differentiable on $J \times \mathbb{R}^{n}$, and that for every fixed $x \in \mathbb{R}^{n} \backslash\{0\}$ the real equation

$$
f(\lambda, x)=0
$$

has at most one solution in $J$. Then equation (3.3) implicitly defines a functional $p$ on some subset $D$ of $\mathbb{R}^{n} \backslash\{0\}$ which replaces the Rayleigh quotient in the variational characterization of eigenvalues of problem (3.1), and which we call the Rayleigh functional.

In the overdamped case, i.e. if $p$ is defined on the entire space $\mathbb{R}^{n} \backslash\{0\}$, the variational characterizations of eigenvalues literally generalize to the nonlinear case. Assume that

$$
x^{T} T^{\prime}(p(x)) x>0 \quad \text { for every } x \neq 0
$$

generalizing the requirement that for symmetric pencils $(K, M)$ a linear combination of the matrices $K$ and $M$ has to be positive definite. Then it holds (cf. [8], [17]) that 
problem (3.3) has $n$ eigenvalues $\lambda_{1} \leq \lambda_{2} \leq \cdots \leq \lambda_{n}$ in $J$, and

$$
\lambda_{k}=\min _{V \in S_{k}} \max _{x \in V, x \neq 0} p(x), \quad k=1,2, \ldots, n .
$$

In the nonoverdamped case $D \neq \mathbb{R}^{n} \backslash\{0\}$ the natural enumeration for which the smallest eigenvalue is the first one, the second smallest is the second one, etc. is not appropriate. This can be easily seen if we make a linear eigenproblem $A x=\lambda x$ nonlinear by reducing the parameter domain $J$ to an interval which does not contain the smallest eigenvalue. Then in general the infimum of the Rayleigh quotient is not an eigenvalue of the reduced problem.

The following enumeration where an eigenvalue $\lambda$ of the nonlinear problem (3.1) inherits its number from the location of the eigenvalue 0 in the spectrum of the matrix $T(\lambda)$ which was introduced in [20] is the key to the minmax characterization in the nonoverdamped case.

If $\lambda \in J$ is an eigenvalue of problem (3.1) then $\mu=0$ is an eigenvalue of the linear problem $T(\lambda) y=\mu y$, and therefore there exists $k \in \mathbb{N}$ such that

$$
0=\max _{V \in S_{k}} \min _{v \in V^{1}} v^{T} T(\lambda) v
$$

where $V^{1}:=\{v \in V:\|v\|=1\}$ is the unit sphere in $V$. In this case we call $\lambda$ a $k$-th eigenvalue of (3.1).

With this enumeration the following minmax characterization of the eigenvalues of the nonlinear eigenproblem (3.1) was proved in [20]. The dual maxmin characterization is contained in [19].

THEOREM 3.1. Assume that for every $x \in \mathbb{R}^{n}, x \neq 0$ the real equation $f(\lambda, x)=$ $x^{T} T(\lambda) x=0$ has at most one solution $p(x)$ in $J$. Let $D$ denote the domain of definition of the Rayleigh functional $p$, and assume that

$$
x^{T} T^{\prime}(p(x)) x>0 \quad \text { for every } x \in D .
$$

Then the following assertions hold:

(i) For every $k \in\{1,2, \ldots, n\}$ there is at most one $k$-th eigenvalue of problem (3.1) in $J$ which can be characterized by

$$
\lambda_{k}=\min _{\substack{V \in S_{k} \\ V \cap D \neq \emptyset}} \sup _{v \in V \cap D} p(v) .
$$

The minimum is attained by the invariant subspace $W$ of $T\left(\lambda_{k}\right)$ corresponding to the $k$ largest eigenvalues of $T\left(\lambda_{k}\right)$, and $\sup _{v \in W \cap D} p(v)$ is attained by all eigenvectors of (3.1) corresponding to $\lambda_{k}$.

(ii) If

$$
\lambda_{k}=\inf _{\substack{V \in H_{k} \\ V \cap D \neq \emptyset}} \sup _{v \in V \cap D} p(v) \in J
$$

for some $k \in\{1, \ldots, n\}$ then $\lambda_{k}$ is the $k$-th eigenvalue of (3.1) and the characterization (3.7) holds.

(iii) If there exists the $k_{1}$-th and the $k_{2}$-th eigenvalue $\lambda_{k_{1}}$ and $\lambda_{k_{2}}$ in $J$ and $k_{1}<k_{2}$, then $J$ contains the $k$-th eigenvalue $\lambda_{k}$ for $k_{1}<k<k_{2}$, as well, and

$$
\inf J<\lambda_{k_{1}} \leq \lambda_{k_{1}+1} \leq \cdots \leq \lambda_{k_{2}}<\sup J
$$


(iv) If $\lambda_{1} \in J$ and $\lambda_{k} \in J$ for some $k$ then every $V \in S_{j}$ with $V \cap D \neq \emptyset$ and $\lambda_{j}=\sup _{u \in V \cap D} p(u)$ is contained in $D$, and the characterization (3.7) can be replaced by

$$
\lambda_{j}=\min _{\substack{V \in S_{j} \\ V^{1} \subset D}} \max _{v \in V^{1}} p(v), \quad j=1, \ldots, k .
$$

4. A priori error bounds. We first consider the component mode synthesis method. With the notations of Section 2 let

$$
\underline{\omega}:=\min \operatorname{diag} \Omega_{1}
$$

be the smallest eigenvalue of problem (2.3) neglected in the CMS method (which can be replaced by the cut off threshold). For $\lambda \in J:=(-\infty, \underline{\omega})$ consider the family of symmetric matrices

$$
T(\lambda)=-\left(\begin{array}{cc}
\Omega_{2} & 0 \\
0 & \tilde{K}_{i i}
\end{array}\right)+\lambda\left(\begin{array}{cc}
I & \tilde{M}_{\ell i 2} \\
\tilde{M}_{i \ell 2} & \tilde{M}_{i i}
\end{array}\right)+\lambda^{2}\left(\begin{array}{c}
0 \\
\tilde{M}_{i \ell 1}
\end{array}\right)\left(\Omega_{1}-\lambda I\right)^{-1}\left(\begin{array}{ll}
0 & \tilde{M}_{\ell i 1}
\end{array}\right) .
$$

Let $\lambda_{1} \leq \lambda_{2} \leq \cdots \leq \lambda_{n}$ denote the eigenvalues of problem (2.1) ordered by magnitude, and let $m \in \mathbb{N}$ such that $\lambda_{m}<\underline{\omega} \leq \lambda_{m+1}$. Then $\lambda_{1}, \ldots, \lambda_{m} \in J$ are the eigenvalues of the nonlinear eigenproblem

$$
T(\lambda) x=0
$$

in $J$. For

$$
f(\lambda, x):=x^{T} T(\lambda) x
$$

it follows from the positive definiteness of $\left(\begin{array}{cc}I & \tilde{M}_{\ell i 2} \\ \tilde{M}_{i \ell 2} & \tilde{M}_{i i}\end{array}\right)$ that

$$
\frac{\partial}{\partial \lambda} f(\lambda, x)=x^{T}\left(\begin{array}{cc}
I & \tilde{M}_{\ell i 2} \\
\tilde{M}_{i \ell 2} & \tilde{M}_{i i}
\end{array}\right) x+\sum_{j=1}^{\nu} \frac{\left(2 \lambda \omega_{j}-\lambda^{2}\right) a_{j}^{2}}{\left(\omega_{j}-\lambda\right)^{2}}>0
$$

for every $x \in \mathbb{R}^{\nu} \backslash\{0\}$. Here $\nu$ denotes the dimension of the reduced problem (2.8), and $a:=\left(\begin{array}{ll}0 & \tilde{M}_{\ell i 1}\end{array}\right) x$.

Hence, for every $x \in \mathbb{R}^{\nu} \backslash\{0\}$ the real equation $f(\lambda, x)=0$ has at most one solution $p(x) \in J$, and condition (3.6) holds. Since $\lambda_{1} \in J$ it follows from Theorem 3.1 that

$$
\lambda_{j}=\min _{\substack{V \in S_{j} \\ V^{1} \subset D}} \max _{x \in V^{1}} p(x) .
$$

The eigenvalues $\tilde{\lambda}_{1} \leq \tilde{\lambda}_{2} \leq \cdots \leq \tilde{\lambda}_{\nu}$ of the reduced problem (2.8) are minmax values of the Rayleigh quotient $R(x)$ corresponding to (2.8), and comparing $p$ and $R$ on appropriate subspaces of $\mathbb{R}^{\nu}$ we arrive at the following bound for the relative errors of the CMS approximations $\tilde{\lambda}_{j}$ to $\lambda_{j}$.

TheOREM 4.1. It holds

$$
0 \leq \frac{\tilde{\lambda}_{j}-\lambda_{j}}{\lambda_{j}} \leq \frac{\lambda_{j}}{\underline{\omega}-\lambda_{j}} \leq \frac{\tilde{\lambda}_{j}}{\underline{\omega}-\tilde{\lambda}_{j}}, \quad j=1, \ldots, m .
$$


Proof. The left inequality, i.e. $\lambda_{j} \leq \tilde{\lambda}_{j}$ is trivial since CMS is a projection method, and the right inequality follows from the monotonicity of the function $\lambda \mapsto \lambda /(\underline{\omega}-\lambda)$.

To prove the inequality in the middle denote by $V \in S_{j}, V \backslash\{0\} \subset D$ the $j$ dimensional subspace of $\mathbb{R}^{\nu}$ such that

$$
\lambda_{j}=\max _{x \in V, x \neq 0} p(x) .
$$

Then $p(x) \leq \lambda_{j}$ for every $x \in V, x \neq 0$, and therefore it follows from (4.5)

$-x^{T}\left(\begin{array}{cc}\Omega_{2} & 0 \\ 0 & \tilde{K}_{i i}\end{array}\right) x+\lambda_{j} x^{T}\left(\begin{array}{cc}I & \tilde{M}_{\ell i 2} \\ \tilde{M}_{i \ell 2} & \tilde{M}_{i i}\end{array}\right) x+\lambda_{j}^{2} x^{T}\left(\begin{array}{c}0 \\ \tilde{M}_{i \ell 1}\end{array}\right)\left(\Omega_{1}-\lambda_{j} I\right)^{-1}\left(\begin{array}{ll}0 & \tilde{M}_{\ell i 1}\end{array}\right) x \geq 0$.

Hence, for every $x \in V, x \neq 0$ one obtains

$$
\lambda_{j} \geq \frac{x^{T}\left(\begin{array}{cc}
\Omega_{2} & 0 \\
0 & \tilde{K}_{i i}
\end{array}\right) x}{x^{T}\left(\begin{array}{cc}
I & \tilde{M}_{\ell i 2} \\
\tilde{M}_{i \ell 2} & \tilde{M}_{i i}
\end{array}\right) x}-\lambda_{j}^{2} \frac{x^{T}\left(\begin{array}{c}
0 \\
\tilde{M}_{i \ell 1}
\end{array}\right)\left(\Omega_{1}-\lambda_{j} I\right)^{-1}\left(0 \begin{array}{ll}
0 & \left.\tilde{M}_{\ell i 1}\right) x
\end{array}\right.}{x^{T}\left(\begin{array}{cc}
I & \tilde{M}_{\ell i 2} \\
\tilde{M}_{i \ell 2} & \tilde{M}_{i i}
\end{array}\right) x} .
$$

In particular for $\hat{x} \in V$ such that $R(\hat{x})=\max _{x \in V, x \neq 0} R(x)$ we have

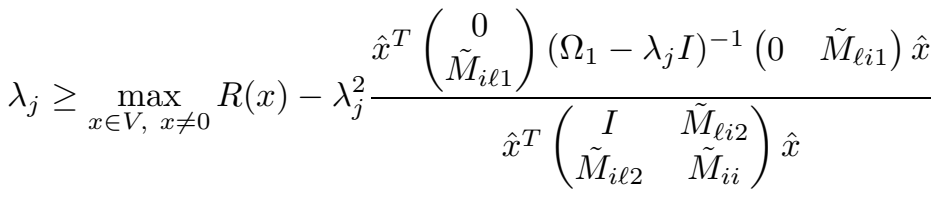

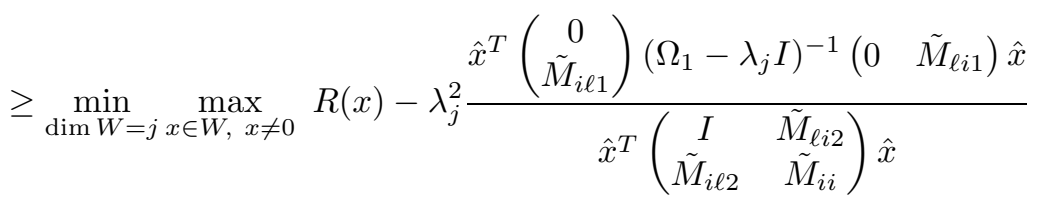

$$
\begin{aligned}
& \geq \tilde{\lambda}_{j}-\frac{\lambda_{j}^{2}}{\underline{\omega}-\lambda_{j}} \max _{x \in \mathbb{R}^{n}, x \neq 0} \frac{x^{T}\left(\begin{array}{c}
0 \\
\tilde{M}_{i \ell 1}
\end{array}\right)\left(\begin{array}{cc}
0 & \tilde{M}_{\ell i 1}
\end{array}\right) x}{x^{T}\left(\begin{array}{cc}
I & \tilde{M}_{\ell i 2} \\
\tilde{M}_{i \ell 2} & \tilde{M}_{i i}
\end{array}\right) x} .
\end{aligned}
$$

From the positive definiteness of the transformed mass matrix

$$
\left(\begin{array}{ccc}
I & 0 & \tilde{M}_{\ell i 1} \\
0 & I & \tilde{M}_{\ell i 2} \\
\tilde{M}_{i \ell 1} & \tilde{M}_{i \ell 2} & \tilde{M}_{i i}
\end{array}\right)
$$

it follows that the Schur complement

$$
\left(\begin{array}{cc}
I & \tilde{M}_{\ell i 2} \\
\tilde{M}_{i \ell 2} & \tilde{M}_{i i}
\end{array}\right)-\left(\begin{array}{c}
0 \\
\tilde{M}_{i \ell 1}
\end{array}\right)\left(\begin{array}{ll}
0 & \tilde{M}_{\ell i 1}
\end{array}\right)
$$

is positive definite as well. Thus,

$$
\max _{x \in \mathbb{R}^{n}, x \neq 0} \frac{x^{T}\left(\begin{array}{c}
0 \\
\tilde{M}_{i \ell 1}
\end{array}\right)\left(\begin{array}{cc}
0 & \tilde{M}_{\ell i 1}
\end{array}\right) x}{x^{T}\left(\begin{array}{cc}
I & \tilde{M}_{\ell i 2} \\
\tilde{M}_{i \ell 2} & \tilde{M}_{i i}
\end{array}\right) x} \leq 1,
$$


and (4.8) yields

$$
\lambda_{j} \geq \tilde{\lambda}_{j}-\frac{\lambda_{j}^{2}}{\underline{\omega}-\lambda_{j}}
$$

which completes the proof.

REMARK 4.1. The special case of Theorem 4.1 for static condensation (2.4) was proved already in [18].

REMARK 4.2. Based on accuracy considerations and an a priori error bound for the smallest eigenvalue (which however usually can not be evaluated since it depends on unknown quantities like a bound for the components of $\tilde{M}_{\ell i 1} \tilde{x}_{3}$ where $\tilde{x}_{3}$ is the interface portion of an eigenvector of (2.6) or the minimal distance of neglected diagonal entries of $\Omega_{1}$ belonging to the same substructure) Yang et al. [21] suggested to neglect all eigenmodes $\left(\omega_{j}, \phi_{j}\right)$ in $(2.7)$ for which

$$
\frac{\lambda_{1}}{\omega_{j}-\lambda_{1}}<\tau
$$

where $\tau \ll 1$ is a small quantity. Theorem 4.1 guarantees that with this choice the relative error of the CMS approximation $\tilde{\lambda}_{1}$ to the smallest eigenvalues $\lambda_{1}$ is less than $\tau$.

For the one level AMLS method (2.14) one obtains with similar arguments as in the proof of Theorem 4.1 the following bounds for the relative errors:

THEOREM 4.2. Let $0<\hat{\lambda}_{1} \leq \hat{\lambda}_{2} \leq \ldots$ be the eigenvalues of the reduced problem (2.14), and denote by $\hat{\mu}$ the smallest eigenvalue of

$$
\left(\begin{array}{cc}
\Omega_{1} & 0 \\
0 & \Gamma_{1}
\end{array}\right) z=\mu\left(\begin{array}{cc}
I & \hat{M}_{12} \\
\hat{M}_{21} & I
\end{array}\right) z
$$

Then it holds

$$
0 \leq \frac{\hat{\lambda}_{j}-\lambda_{j}}{\lambda_{j}} \leq \frac{\lambda_{j}}{\hat{\mu}-\lambda_{j}}
$$

for every eigenvalue $\lambda_{j}$ of (2.1) such that $\lambda_{j}<\hat{\mu}$.

Proof. In the same way as in the proof of Theorem 4.1 we obtain from (2.13), $(2.14)$, and $(2.15)$

$$
\begin{gathered}
\lambda_{j} \geq \hat{\lambda}_{j}-\lambda_{j}^{2} \max _{x \neq 0} \frac{x^{T}\left(\begin{array}{cc}
0 & \hat{M}_{32} \\
\hat{M}_{41} & 0
\end{array}\right)\left(\begin{array}{cc}
\Omega_{1}-\lambda_{j} I & -\lambda_{j} \hat{M}_{12} \\
-\lambda_{j} \hat{M}_{21} & \Gamma_{1}-\lambda_{j} I
\end{array}\right)^{-1}\left(\begin{array}{cc}
0 & \hat{M}_{14} \\
\hat{M}_{23} & 0
\end{array}\right) x}{x^{T}\left(\begin{array}{cc}
I & \hat{M}_{34} \\
\hat{M}_{43} & I
\end{array}\right) x} \\
\geq \hat{\lambda}_{j}-\frac{\lambda_{j}^{2}}{\hat{\mu}-\lambda_{j}} \max _{x \neq 0} \frac{x^{T}\left(\begin{array}{cc}
0 & \hat{M}_{32} \\
\hat{M}_{41} & 0
\end{array}\right)\left(\begin{array}{cc}
I & \hat{M}_{12} \\
\hat{M}_{21} & I
\end{array}\right)^{-1}\left(\begin{array}{cc}
0 & \hat{M}_{14} \\
\hat{M}_{23} & 0
\end{array}\right) x}{x^{T}\left(\begin{array}{cc}
I & \hat{M}_{34} \\
\hat{M}_{43} & I
\end{array}\right) x},
\end{gathered}
$$

and the positive definiteness of the transformed mass matrix in (2.13) yields that the fraction on the right is less than or equal to 1. 
A severe disadvantage of Theorem 4.2 is the fact the bound depends on the smallest eigenvalue of problem (4.10), i.e. on the Matrix

$$
\hat{M}_{12}=\Phi_{1}^{T}\left(M_{\ell i}-M_{\ell \ell} K_{\ell \ell}^{-1} K_{\ell i}\right) \Psi_{1}
$$

which usually is not at hand because it is much too expensive to determine the eigenmodes of problems (2.5) and (2.10) corresponding to eigenvalues greater than the cut off bound. The following Theorem 4.3 does not suffer this drawback.

THEOREM 4.3. Let $\underline{\omega}$ and $\underline{\gamma}$ be the smallest entry of $\Omega_{1}$ and $\Gamma_{1}$, respectively. Then it holds

$$
\begin{aligned}
0 \leq \frac{\hat{\lambda}_{j}-\lambda_{j}}{\lambda_{j}} & \leq \frac{\hat{\lambda}_{j}}{\underline{\gamma}-\hat{\lambda}_{j}}+\frac{\lambda_{j}}{\underline{\omega}-\lambda_{j}}\left(1+\frac{\hat{\lambda}_{j}}{\underline{\gamma}-\hat{\lambda}_{j}}\right) \\
& \leq \frac{\hat{\lambda}_{j}}{\underline{\gamma}-\hat{\lambda}_{j}}+\frac{\hat{\lambda}_{j}}{\underline{\omega}-\hat{\lambda}_{j}}+\frac{\hat{\lambda}_{j}^{2}}{\left(\underline{\omega}-\hat{\lambda}_{j}\right)\left(\underline{\gamma}-\hat{\lambda}_{j}\right)}
\end{aligned}
$$

for every eigenvalue $\lambda_{j}$ of $(2.1)$ such that $\lambda_{j}<\min \{\underline{\omega}, \underline{\gamma}\}$.

Proof. To prove the inequality (4.12) we generate the reduced problem (2.14) in two CMS reduction steps, first dropping eigenmodes of problem (2.4) to obtain problem (2.8), and then applying CMS to this problem neglecting eigenmodes of (2.10). Inequality (4.13) then follows immediately by a monotonicity argument.

Let $\tilde{\lambda}_{j}$ be the eigenvalues of problem (2.8). Then it follows from (4.9)

$$
\lambda_{j} \geq \tilde{\lambda}_{j}-\frac{\lambda_{j}^{2}}{\underline{\omega}-\lambda_{j}}
$$

and applying (4.9) to the CMS reduction of (2.8) to (2.14) we obtain

$$
\tilde{\lambda}_{j} \geq \hat{\lambda}_{j}-\frac{\tilde{\lambda}_{j}^{2}}{\underline{\gamma}-\tilde{\lambda}_{j}}
$$

Hence,

$$
\hat{\lambda}_{j} \geq \tilde{\lambda}_{j}\left(1+\frac{\tilde{\lambda}_{j}}{\underline{\gamma}-\tilde{\lambda}_{j}}\right) \geq \lambda_{j}\left(1+\frac{\lambda_{j}}{\underline{\omega}-\lambda_{j}}\right)\left(1+\frac{\tilde{\lambda}_{j}}{\underline{\gamma}-\tilde{\lambda}_{j}}\right)
$$

from which we immediately obtain (4.12).

The proof of Theorem 4.3 suggests how to obtain an a priori bound for the general AMLS method. Every reduction step obtaining a quasistatic/modal representation and reducing the dimension by spectral truncation is identical to a CMS step utilizing the substructuring of the next level. Hence, if $\lambda_{j}^{(\nu)}$ denotes the eigenvalues of the reduced eigenvalue problem corresponding to the $\nu$-th level ordered by magnitude, if $\lambda_{j}^{(0)}:=\lambda_{j}$, and $\lambda_{j}^{(p+1)}$ are the eigenvalues of the projected eigenproblem of AMLS with $p$ levels of substructuring, where on the $\nu$-th level eigenvalues exceeding $\omega_{\nu}$ are neglected, then it holds by (4.9)

$$
\lambda_{j}^{(\nu)} \leq \lambda_{j}^{(\nu-1)}\left(1+\frac{\lambda_{j}^{(\nu-1)}}{\omega_{\nu}-\lambda_{j}^{(\nu-1)}}\right), \quad \nu=1,2, \ldots, p+1 .
$$




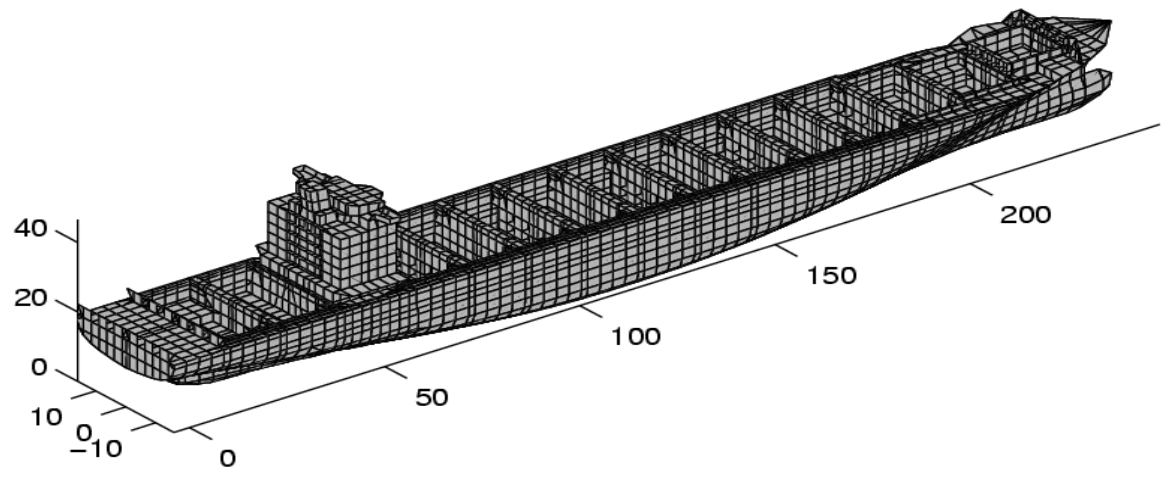

Fig. 1. FE model of a container ship

Thus, it follows for all $\lambda_{j} \leq \min _{\nu=1, \ldots, p} \omega_{\nu}$

$$
\lambda_{j}^{(p+1)} \leq \lambda_{j} \prod_{\nu=0}^{p}\left(1+\frac{\lambda_{j}^{(\nu)}}{\omega_{\nu+1}-\lambda_{j}^{(\nu)}}\right)
$$

THEOREM 4.4. Let

$$
\tilde{\lambda}_{1} \leq \tilde{\lambda}_{2} \leq \tilde{\lambda}_{m}<\min _{\nu=0, \ldots, p} \omega_{\nu} \leq \tilde{\lambda}_{m+1} \leq \ldots
$$

be the eigenvalues of the projected eigenproblem by AMLS with p levels of substructuring where on the $\nu$-th level eigenvalues exceeding $\omega_{\nu}$ are neglected. Then it holds

$$
\frac{\tilde{\lambda}_{j}-\lambda_{j}}{\lambda_{j}} \leq \prod_{\nu=0}^{p}\left(1+\frac{\lambda_{j}^{(\nu)}}{\omega_{\nu}-\lambda_{j}^{(\nu)}}\right)-1, \quad j=1, \ldots, m .
$$

5. Numerical experiments. To verify the quality of our a priori bounds we considered the vibrational analysis of a container ship which is shown in Figure 1. Usually in the dynamic analysis of a structure one is interested in the response of the structure at particular points to harmonic excitations of typical forcing frequencies. For instance in the analysis of a ship these are locations in the deckshouse where the perception of the crew is particularly strong.

The finite element model of the ship (a complicated 3 dimensional structure) is not meshed with an automatic preprocessor like ANSYS or PATRAN since this would result in a much too detailed model. Since bending displacements of the plates do not influence the displacements in the deckhouse for global vibrations, it suffices to discretize the surface by linear membrane shell elements with additional truss elements for the stiffeners. Only the engine is modelled with more detail. For the ship under consideration this yields a very coarse model with 19106 elements and 12273 nodes resulting in a discretization with 35262 degrees of freedom.

We consider the structural deformation caused by a harmonic excitation at a frequency of $4 \mathrm{~Hz}$ which is a typical forcing frequency stemming from the engine and 


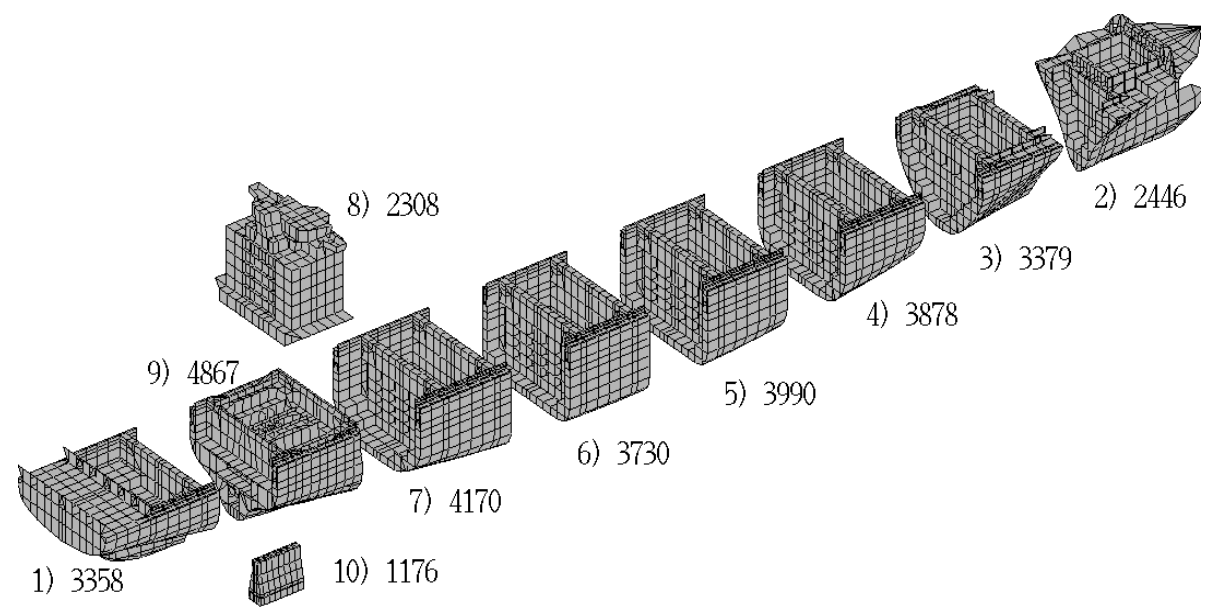

Fig. 2. Substructuring

the propeller. Since the deformation is small the assumptions of the linear theory apply, and the structural response can be determined by the mode superposition method taking into account eigenfrequencies in the range between 0 and $7.5 \mathrm{~Hz}$ (which corresponds to the 50 smallest eigenvalues for the ship under consideration).

To apply the CMS method and the level 1 version of AMLS we partitioned the FEM model into 10 substructures as shown in Figure 2. This substructuring by hand yielded a much smaller number of interface degrees of freedom than automatic graph partitioners which try to construct a partition where the substructures have nearly equal size. For instance, our model ends up with 1960 degrees of freedom on the interfaces, whereas Chaco [11] ends up with a substructuring into 10 substructures with 4985 interface degrees of freedom.

We solved the eigenproblem by the CMS method using a cut off bound of 20,000 (about 10 times the largest wanted eigenvalue $\lambda_{50} \approx 2183$ ). 329 eigenvalues of the substructure problems were less than our threshold, and the dimension of the resulting projected problem was 2289. Figure 3 shows the relative errors for the smallest 50 eigenvalues (lower crosses) and the error bounds by Theorem 4.1 (upper crosses). We reduced the interface degrees of freedom as well with the same cut off bound 20,000. This reduced the dimension of the projected eigenproblem to 436 . The relative errors (lower circles) and bounds by Theorem 4.3 are shown in Figure 3, too.

We substructured the FE model by Metis with 4 levels of substructuring. Neglecting eigenvalues exceeding 20,000 and 40,000 on all levels AMLS produced a projected eigenvalue problem of dimension 451 and 911, respectively. The relative errors and the bounds are shown in Figure 5 where the lower and upper crosses correspond to the threshold 20,000, and the lower and upper circles to 40,000.

Acknowledgements. Thanks are due to Christian Cabos, Germanischer Lloyd, who provided us with the finite element model of the container ship. The first author gratefully acknowledges financial support of this project by the German Foundation of Research (DFG) within the Graduiertenkolleg "Meerestechnische Konstruktionen".

\section{REFERENCES}

[1] Z. Bai and B.-S. Lia. Towards an optimal substructuring method for model reduction. Technical 


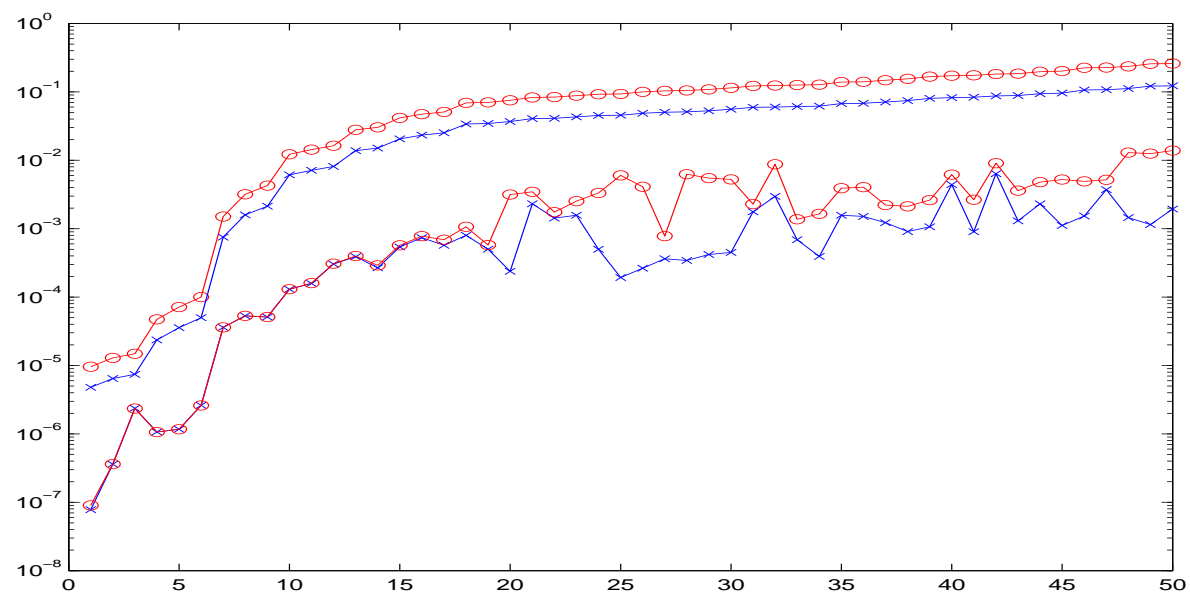

Fig. 3. Errors and bounds for CMS

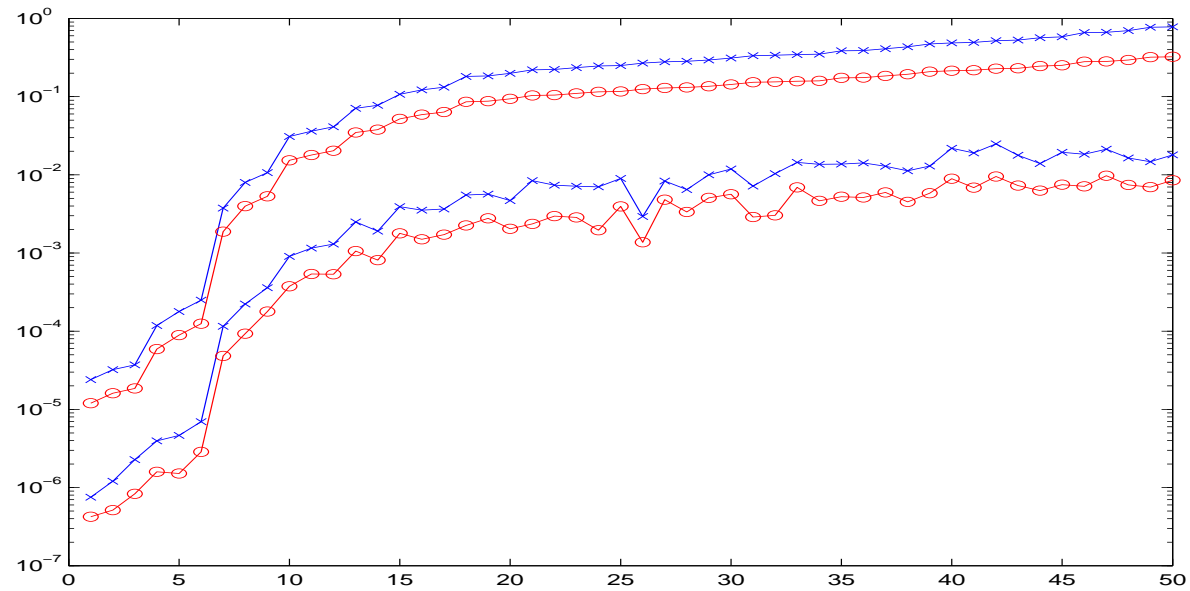

Fig. 3. Errors and bounds for AMLS

report, University of California at Davis, 2004. To appear in Proceedings of PARA'04, Lyngby, Denmark, 2004.

[2] C. Bekas and Y. Saad. Computation of smallest eigenvalues using spectral Schur complements. Technical Report umsi-2003-191, Minnesota Supercomputer Institute, University of Minnesota, Minneapolis, 2004.

[3] J.K. Bennighof and M.F. Kaplan. Frequency sweep analysis using multi-level substructuring, global modes and iteration. In Proceedings of the AIAA 39th SDM Conference, Long Beach, Ca., 1998.

[4] J.K. Bennighof, M.F. Kaplan, M.B. Muller, and M. Kim. Meeting the NVH computational challenge: automated multi-level substructuring. In Proceedings of the $18^{\text {th }}$ International Modal Analysis Conference, San Antonio, Texas, 2000.

[5] J.K. Bennighof and C.K. Kim. An adaptive multi-level substructuring method for efficient modeling of complex structures. In Proceedings of the AIAA 33rd SDM Conference, pages 1631 - 1639, Dallas, Texas, 1992.

[6] J.K. Bennighof and R.B. Lehoucq. An automated multilevel substructuring method for the eigenspace computation in linear elastodynamics. SIAM J. Sci. Comput., 25:2084-2106, 2004.

[7] R.R. Craigh Jr. and M.C.C. Bampton. Coupling of substructures for dynamic analysis. AIAA J., 6:1313-1319, 1968.

[8] R.J. Duffin. A minmax theory for overdamped networks. J.Rat.Mech.Anal., 4:221 - 233, 1955. 
[9] K. Elssel and H. Voss. A modal approach for the gyroscopic quadratic eigenvalue problem. In R. Neittaanmïi, T. Rossi, S. Korotov, E. Onate, J. Periaux, and D. Knörzer, editors, Proceedings of the European Congress on Computational Methods in Applied Sciences and Engineering. ECCOMAS 2004, Jyväskylä, Finland, 2004. ISBN 951-39-1869-6.

[10] R. J. Guyan. Reduction of stiffness and mass matrices. AIAA J., 3:380, 1965.

[11] B. Hendrickson and R. Leland. The Chaco User's Guide: Version 2.0. Technical Report SAND94-2692, Sandia National Laboratories, Albuquerque, 1994.

[12] W.C. Hurty. Vibration of structure systems by component-mode synthesis. J.Engrg.Mech.Div., $A S C E, 86: 51-69,1960$.

[13] B. Irons. Structural eigenvalue problems: Elimination of unwanted variables. AIAA J., 3:961962, 1965.

[14] M.F. Kaplan. Implementation of Automated Multilevel Substructuring for Frequency Response Analysis of Structures. PhD thesis, Dept. of Aerospace Engineering \& Engineering Mechanics, University of Texas at Austin, 2001.

[15] G. Karypis and V. Kumar. Metis. a software package for partitioning unstructured graphs, partitioning meshes, and computing fill-reducing orderings of sparse matrices. version 4.0. Technical report, University of Minnesota, Minneapolis, 1998.

[16] A. Kropp and D. Heiserer. Efficient broadband vibro-acoustic analysis of passenger car bodies using an FE-based component mode synthesis approach. In H.A. Mang, F.G. Rammerstorfer, and J. Eberhardsteiner, editors, Proceedings of the Fifth World Congress on Computational Mechanics (WCCM V), Vienna, Austria, 2002. available online from http://wccm.tuwien.ac.at.

[17] E.H. Rogers. A minmax theory for overdamped systems. Arch.Rat.Mech.Anal., 16:89 - 96, 1964.

[18] H. Voss. An error bound for eigenvalue analysis by nodal condensation. In J. Albrecht, L. Collatz, and W. Velte, editors, Numerical Treatment of Eigenvalue Problems, Vol. 3, volume 69 of International Series on Numerical Mathematics, pages 205-214, Basel, 1984. Birkhäuser.

[19] H. Voss. A maxmin principle for nonlinear eigenvalue problems with application to a rational spectral problem in fluid-solid vibration. Applications of Mathematics, 48:607 - 622, 2003.

[20] H. Voss and B. Werner. A minimax principle for nonlinear eigenvalue problems with applications to nonoverdamped systems. Math.Meth.Appl.Sci., 4:415-424, 1982.

[21] C. Yang, W. Gao, Z. Bai, X. Li, L. Lee, P. Husbands, and E. Ng. An algebraic sub-structuring method for large-scale eigenvalue calculations. Technical Report LBNL-55050, Lawrence Berkeley National Laboratory, Berkeley, Ca., 2004. 